\title{
COMMENT LE TÉMOIGNAGE DEVIENT UNE CEUVRE LITTÉRAIRE : LE CAS DE ROBERT ANTELME ET DE PRIMO LEVI
}

\begin{abstract}
Teklik Joanna, Comment le témoignage devient une ozuvre littéraire: Le cas de Robert Antelme et de Primo Levi [How testimony becomes a literary work: thc case of Robert Antelme and Primo Levi]. Studia Romanica Posnaniensia, Adam Mickiewicz University Press, Poznan, vol. XXXIIl : 2006, pp. 49-63. ISBN 83-232-1643-6, ISSN 0137-2475.
\end{abstract}

Can a testimony, a text similar to a document, be a literary work? The answer to this question is definitely positive in the case of R. Antelme ( $L$ 'espèce humaine) and P. Levi (Se questo è un uomo) who relate their experience from a concentration camp. These authors, remaining faithful to the truth, do not neglect aesthetic requirements, using known narrative techniques and skillfully maintaining the tension characteristic of literary texts. They use an innovative form of narration. It is fragmentary and broken, which fully reflects the character of the prisoners' fate. Finally, a new conception of humanity and a deep reflection on human nature (the titles are meaningful themselves) prove the literary character of both texts.

De nombreux ouvrages publiés après l'année 1945 portent l'étiquette de littérature concentrationnaire. Dans la plupart des cas il est question des documents qui nous renvoient au vécu des camps de concentration et de la déportation même. Ces textes, transcrits d'une manière plus ou moins engagée, témoignent souvent de l'impuissance de la littérature face à la représentation de l'horreur. Il serait intéressant d'analyser dans le cadre de cet article deux témoignages importants qui peuvent particulièrement attirer l'attention du lecteur, à savoir $L$ 'Espèce humaine de Robert Antelme et Se questo è un uomo de Primo Levi. Leurs auteurs recourent d'une façon originale aux traumatismes liés à leur déportation et dépassent les conventions propres au témoignage perçu dans un sens habituel, c'est-à-dire comme une simple déclaration de ce qu'on a vu ou entendu.

Afin de pouvoir mieux en juger, il faudrait cependant se rappeler en premier lieu le caractère et la spécificité des textes testimoniaux parus durant l'après-guerre. Ils restent toujours problématiques pour les critiques littéraires qui préfèrent rajouter cet adjectif qualificatif concentrationnaires non seulement pour mettre ainsi en 
relief leur origine, mais aussi pour marquer la distance qui les sépare de la littérature. Les chercheurs, à tort ou à raison, refusent de reconnaître la « littérarité » de ces écrits, persuadés que l'expérience d'un déporté est incapable de donner naissance à une œuvre d'art. A leurs yeux, les témoignages se concentrent avant tout sur le message transmis, tout en privilégiant la fonction de communication (au sens jacobsonien du terme), et, par conséquent, ils ne répondent pas aux exigences esthétiques.

Il n'est donc point étonnant que la question de la mise en forme du vécu concentrationnaire, discutée d'ailleurs largement dans les cercles littéraires de l'aprèsguerre', et par les rescapés eux-mêmes ${ }^{2}$, revient d'une manière spectaculaire durant les quinze dernières années. Le regain d'intérêt est d'autant plus considérable si l'on regarde le nombre d'ouvrages consacrés récemment à cette problématique. Les chercheurs évoquent, à maintes reprises et dans contextes différents, le paradoxe propre aux textes en question : ils entendent témoigner d'une expérience collective qui resterait en définitive incommunicable à ceux qui ne l'auraient pas partagée. Ceci les amène par la suite à se demander pourquoi la fiction rendrait l'événement « transmissible » et en quoi consiste cette impossibilité du témoignage.

Dans un article intitulé Le témoignage et la fiction, Bruno Gelas ${ }^{3}$ cherche à élucider ce problème, en recourant principalement à la notion de fiction, telle que la conçoit Grenette ${ }^{4}$. L'analyse du fonctionnement des deux termes amène l'auteur à reconsidérer le problème du rapport qu'ils entretiennent avec les catégories du vrai et du faux. La fiction littéraire, dit Gelas, n'est pas vraie et elle ne renvoie, dans l'ordre de la réalité, à rien. Elle est cette « dénotation sans dénoté » (Genette) qui ne se fonde sur rien. C'est à partir de cette absence qu'elle commence et acquiert du sens. Elle se soustrait, souligne l'auteur, à l'envahissement par l'événement et à l'asservissement à l'authenticité des faits, qui forment la condition et le malheur du

${ }^{1}$ La littérature n'est pas préparée à ce genre de compte rendu, déclarent les uns, et les événements d'Auschwitz, ne supporteraient certainement pas une description à caractère littéraire (Cf. B. B recht, Ecrits sur la politique ef la société, L'Arche, Paris 1970, p. 244). L'écriture en tant que tclle, soutiennent les autres, risque de violer l'expérience et la transmission de la vérité dans le cadre d'une composition «esthétique" semble d'emblée vorée à l'échec. Th. Adorno observe par exemple que lorsqu'on parle des choses extrêmes, on ćprouve une sorte de honte à l'égard de la forme, comme si celle-ci faisait outrage à la souffrance, en la réduisant impitoyablement à l'état d'un matériau mis à sa disposition. Cf. Th. Adorno, Modeles critiques, Payot, Paris 1984, p. 135.

${ }^{2}$ Selon Jean Cayrol, l'arrangement fictif des événements risque de transformer ce vécu singulier en une inage fixe et dépassionné. Ceci dit, le mécanisme de la fictionnalisation peut être conçu comme la mise en doute de la véracité de l'expérience. Cependant, Semprun et ses camarades constatent que la vérité essentielle de l'expérience n'est transmissible que par l'écriture littéraire. Il faudrait une fiction, dit-il, mais qui osera? Cf. J. Cayrol, Témoignage et littérature, Esprit, avril 1953, p. 575-578 et J. Semprun, L'Ecriture ou la vie, Gallimard, Paris 1994, p. 138.

${ }^{3}$ Cf. Les Cahiers de la Villa Gillet, $\mathrm{n}^{\circ} 3$, novembre 1995, Le témoignage : éthique, esthétique et pragmatique (II), pp. 61-68.

${ }^{4}$ Pour la terminologie, cf. G. Genette, Fiction et diction, Seuil, Paris 1991. 
témoignage. Comme la fiction renvoie d'emblée à l'imaginaire, l'irréel, la question de la vérité transmise n'y apparaît plus. Il en découle une sorte d'irresponsabilité qui s'installe entre l'auteur et son récepteur, ce qui rend sans doute le processus même de «transmission », moins complexe pour le survivant. En cherchant à s'établir dans la fiction, les rescapés se libèrent de leur souci principal, tant soulevé à des occasions différentes, qui est la véracité de leur témoignage. Ainsi, ils franchissent plus facilement la frontière de l'indicible, entendu comme une divergence entre un vécu personnel «limite » et son expression verbale ${ }^{5}$.

Les deux récits - Se questo è un uomo de Primo Levi et L'espèce humaine de Robert Antelme - ne sont pas de simples témoignages, puisque les auteurs font appel à la technique romanesque et maintiennent dans leur ouvre la tension propre aux ouvrages littéraires. Ils souscrivent par là à l'opinion des auteurs anglo-saxons selon laquelle il faut considérer l'écriture comme un acte où la conscience s'oppose à la pression de la réalité épouvantable ${ }^{6}$. La littérature conçue de cette manière devient la force qui agit de l'intérieur, tout en nous protégeant contre la violence de l'extérieur. Elle est donc l'imagination qui fait face à la pression de la réalité ${ }^{7}$. Tel est le cas de ces récits que l'on pourrait définir comme «témoignages littéraires». Afin de saisir la signification de ce terme un peu paradoxal, M. Riffaterre propose de commencer par distinguer le témoignage de sa représentation. Si l'on admet que le témoignage, comme le remarque l'auteur ${ }^{8}$, est l'acte de se porter garant de l'authenticité de ce que l'on observe et qu'on croit digne d'être rapporté, le témoignage littéraire est alors la représentation de cet acte authentique, et de l'objet qu'il authentifie, dans une œuvre d'art verbal qui leur confère sa littérarité. Ceci dit, la littérarité ajoute au témoignage l'effet de réel qui, dans la transcription de la réalité concentrationnaire n'y est pas sans importance. Les textes de Levi et d'Antelme poursuivent en quelque sorte le chemin indiqué par Chateaubriand ou Hugo, deux observateurs engagés de leur époque, qui ont créé une écriture poétique inspirée de leur témoignage.

${ }^{5}$ Le concept de l'indicible est largement élaboré par M. Rinn, dans son ouvrage Les récits du génocide. Sémiotique de l'indicible. Delachaux et Niestlé S.A., Lausanne - Paris 1998. Pour la définition, voir p. 7 .

${ }^{6}$ Cf. à ce sujet : A.H. Rosenfeld, Podwójna śmierć. Rozważania o literaturze Holocaustu. Wydawnictwo Cyklady, Warszawa 2003, p. 108.

${ }^{7}$ Cette optique va à l'encontre de celle que propose par exemple Michèle Rosellini. Elle soutient que le récit est impossible, non par sa matière et sa réception, mais par les sentiments qu'éprouvent les survivants au moment de le rédiger. En tant que premiers auditeurs de leur récit, ils prennent conscience de l'abîme qui sépare le texte et l'événement qu'il est censé relater. Cf. son article intitulé Entre fiction et l'expérience : le lecteur de L'Espèce humaine, dans : Les camps et la littérature. Une littérature du $X X^{c}$ siècle, D. Dobbels et D. Moncond'huy (dir.), La licorne, Poitiers 1999, pp. 159-182.

${ }^{8} \mathrm{Cf}$. M. Riffaterre, Le témoignage littéraire, Les Cahiers de la Villa Gillet, $\mathrm{n}^{\mathrm{b}} 3$, novembre 1995, op. cit., pp. 33-55. 
En outre, les deux récits se placent sans doute dans l'authenticité autobiographique ${ }^{9}$, puisque leurs auteurs ne cherchent pas à révéler un savoir général sur le Lager, mais répondent à l'exigence de fidélité et de sincérité. Ainsi, ils relatent non seulement les événements réellement vécus, mais ceux qui ont été vécus de l'intérieur du camp. D'autre part, contrairement à la simple autobiographie, à l'authenticité des textes de Levi et d'Antelme, s'ajoute la vérité de leur témoignage, ce qui déplace également l'expérience concentrationnaire dans le champ de l'expérience littéraire.

A côté de ce rapport singulier à la vérité, on peut trouver d'autres critères qui permettraient de distinguer entre le simple témoignage sur les camps, un document brut à une charge émotionnelle considérable et l'œuvre littéraire où l'exigeance esthétique prend le pas sur le message transmis. Yves Stalloni, par exemple, dans un de ses articles récents, parle de deux autres références importantes, l'invention d'une forme et la visée universelle du texte ${ }^{10}$.

Il faut bien l'admettre, une telle expérience nécessite une écriture et une forme nouvelles qui, tout en rompant avec la cohérence narrative habituelle, sont appelées à rendre la «désorganisation » de l'existence du détenu. D'où le goût pour la peinture fragmentaire du vécu concentrationnaire, propre aux témoignages des survivants. Le fragment devient dans ces textes la forme la plus appropriée peut-être, celle qui permet de rendre de manière significative la dégradation de l'homme, sa souffrance physique et morale, ainsi que l'indicible de son expérience. L'écriture fragmentaire répond ainsi à la représentation de la réalité de l'après-guerre, vu la négation du monde qu'elle implique, la contestation de la totalité et de la cohérence. Dans ce contexte, on pourrait inscrire la remarque faite par P. Garrigues ${ }^{11}$. Le fragment et la fragmentation, dit-il, ne prétendent pas nier la vie en tant que telle, mais plutôt ses interprétations globalisantes, scientifiques. Cette négation veut détruire le mythe d'une transparence du monde au langage qui le signifierait dans sa totalité. Outre un refus, il faut y donc voir la recherche de quelque chose d'authentique dans les relations entre l'homme et le monde, le langage et le silence, une sorte de rajustement entre les mots et les choses.

L'écriture fragmentaire qui conduit à contester la totalité devient par là une écriture de médiation. Elle ne traduit le monde que partiellement et se situe entre l'ouverture et la fermeture. Cette position «à mi-chemin» permet de mieux voir la complexité de la problématique abordée. A la manière dialectique parfois, deux

${ }^{9} \mathrm{~L}$ 'authenticité, selon la définition proposée par $\mathrm{Ph}$. Lejeune, traduit ce rapport intérieur propre à l'emploi de la première personnne dans le récit personnel et il na faut pas le confondre ni avec l'didentité qui renvoie au nom propre, ni avec la ressemblance, qui suppose un sentiment de similitude entre deux images différentes porté par une tierce personne. Cf. $\mathrm{Ph}$. Leje une, Le pacte autobiographique, Seuil, p. 40.

${ }^{10} \mathrm{Cf}$. Le magazine littéraire, $\mathrm{n}^{\circ} 438$, janvier 2005 , consacré à la littérature et les camps, et plus particulièrement, l'article de Y. Stalloni, intitulé De l'horreur à la littérature, pp. 41-44.

${ }^{1}$ Cf. P. Garrigues, Poétiques du fragment, Klincksieck, Paris 1995, p. 122. 
contradictions se rencontrent et contribuent à formuler une synthèse. Le fragmentaire exprime, par définition même, la contrariété (un par rapport à tout) et peut aussi mener à révéler toute une suite d'oppositions, telles par exemple vie/mort, etc $^{12}$.

Ceci ne reste pas sans lien avec la visée universelle du texte, mentionnée plus haut, car de la vision fragmentaire du monde découle également la nouvelle perception de la nature humaine. La méditation sur l'identité de l'homme, mis à rudes épreuves fait naître dans les textes étudiés une vraie idéologie de l'homme. L' individu-témoin c'est souvent un homme qui devient ce qu'il n'est pas, qui revient à sa nature animale et spécifique, qui la contredit finalement pour atteindre une « autre nature». Il représente ainsi toute l'humanité souffrante, frappée par l'horreur de la détention.

Voyons maintenant comment les auteurs de Se questo è un uomo et de L'espèce humaine cherchent à atteindre lesdits postulats de littérarité à travers leurs récits. Si similaire que soit leur message sur la condition du déporté, la manière dont ils réalisent leurs objectifs, la démarche qu'ils choisissent, ainsi que la conclusion qu'ils formulent, restent pourtant différentes. Tout d'abord le passage même à l'écriture s'effectue autrement chez les deux déportés. Antelme se met à écrire avant son arrestation et il a déjà quelques notions de littérature au sens traditionnel du mot. Cela implique ses hésitations et réserves face à la rédaction d'un texte qui exige d'emblée une démarche spécifique et presque inconnue jusqu'alors. Responsable et soucieux, il essaie de transcrire son vécu par un langage approprié.

Chez Levi, chimiste de formation, on ne peut point parler de préoccupation littéraire quelconque, ce n'est qu'après sa déportation qu'il naît en tant qu'écrivaintémoin. Contrairement à Antelme, il ne se soucie pas trop de la forme de son témoignage dans le sens où il est loin de toute recherche d'ordre esthétique. «I problemi di stile mi sembravano ridicoli, dit-il, (...) mi pareva, questo libro, di averlo già in testa tutto pronto, di doverlo solo lasciare uscire e scendere sulla carta $)^{13}$. Bien qu'il refuse la littérature qui est pour lui le synonyme de fiction, d'invention, ceci ne ferme pas, comme nous allons voir plus loin, la question du rapport du témoignage levien à la littérature. Si ces deux récits peuvent être considérés comme des œuvres littéraires, ce n'est pas par une parenté quelconque avec le roman, mais par le travail spécifique sur l'énonciation que leurs auteurs réalisent.

${ }^{12}$ Cette optique correspond à l'approche de M. Blanchot qui parle de l'expérience concentrationnaire, tout en focalisant sa réflexion sur $L$ 'espèce humaine. Pour lui, la pensće fragmentaire reste la pensée de la vie. Or, it est ici question de la vie qui ne pourrait se penser que dans la mort, quelle que soit sa forme (la mort du langage, de la pensée ou de la réalité). Blanchot analyse le témoignage de R. Antelme par le truchement d'un renversement de perspective, nécessaire à comprendre la situation de l'homme et de son épreuve. Cf. M. Blanchot, L'entretien infini, Gallimard. Paris 1969, pp. 191-200.

${ }^{13} \mathrm{C}$. l'appendice à Se questo è un uomo, écrit cn novembre 1976, pour la citation voir p. 349. 
Les attitudes de Levi et d'Antelme à l'égard de l'écriture se rejoignent au moment où ils décident de transcrire leur vécu. Avant de nous faire connaître la réalité concentrationnaire, ils annoncent déjà par le biais des péritextes (l'avantpropos pour Antelme et la préface pour Levi) les problèmes de base qu'implique leur double expérience, d'abord celle du déporté, ensuite celle d'écrivain-témoin. Dès le début ces auteurs situent la véridicité de leurs témoignages hors de doute : « je rapporte ici ce que $\mathrm{j}$ 'ai vécu, dit Antelme dans son avant-propos, nessuno dei fatti è inventato », affirme Levi dans sa préface.

Dans un premier mouvement, l'auteur de L'espèce humaine commence par s'identifier à tous les déportés. C'est à la première personne du pluriel qu'il évoque le moment difficile du retour, moment crucial car le sentiment de parler, d'être entendu enfin s'empare de lui et des autres détenus. Ce sentiment est justifié sans doute si l'on considère le camp comme la réfutation radicale de tout principe de communication obligatoire (ce phénomène du non-parlé est présent dans le texte). Le besoin de communiquer leur expérience devient donc prioritaire, mais une fois formulé, il suscite quelques doutes. Antelme se demande au nom de tous les détenus s'ils sont capables de combler cet abîme entre ce qu'ils ont vécu et le récit qu'il est possible d'en faire. S'il est question d'une réalité qui dépasse l'imagination, alors c'est encore par l'imagination, comme le souligne l'auteur, qu'ils peuvent en dire quelque chose. Son témoignage reflète donc encore une fois cet aspect paradoxal des textes nés autour des camps de concentration. De manière dialectique aussi apparaît la volonté de faire comprendre ce que l'on ne peut pourtant pas comprendre, exprimer l'inimaginable grâce à un surcroît d'imagination, et pouvoir enfin dire l'indicible. Dès le début le lecteur peut ressentir une angoisse étrange à l'égard du phénomène, qui, étant indescriptible par l'homme, relève peut-être du domaine de l'inhumain ${ }^{14}$.

Ce n'est que plus tard que surgit dans le discours préfaciel le « je » de l'auteur. Il précise son projet d'esquisser la vie d'un kommando de Gandersheim et de son appareil administratif, pour mettre ainsi en relief les différences qui existent entre les autres camps ayant pour responsables les déportés politiques. Une fois la problématique signalée, l'auteur formule son objectif: d'abord celui du déporté voué au combat individuel pour survivre, rester jusqu'au bout un homme, ensuite celui du héros de son propre témoignage amené, contrairement aux héros littéraires au sens traditionnel du mot, à "exprimer comme seule et dernière revendication un sentiment ultime d'appartenance à l'espèce » $(\mathrm{EH}, 11)$. C'est ainsi qu'Antelme nous prépare à comprendre son message principal qui est "l'expérience-limite » de l'homme $^{15}$. La mise en question de la qualité d'homme déclenche donc toute une

${ }^{14} \mathrm{Cf}$. L. Pipet, La notion d'indicible dans la littérature des camps de la mort. L'Harmattan, Paris 2000 , p. 26.

${ }^{15}$ On doit cette expression à M. Blanchot qui particulic̀rement intéressé à l'écriture d'Antelme, lui consacre quelques pages dans le chapitre intitulé "Expérience - limite" de son Entretien infini. Gallimard, Paris 1969, pp. 191-200. 
réflexion autour des limites de l'espèce humaine, de sa complexité et de sa solitude. Cette réflexion, comme le constate l'auteur à la fin de son avant-propos, ne sert qu'à mieux concevoir l'unité indivisible de cette espèce.

Quand on analyse la préface à Se questo è un uomo, on aperçoit quelques éléments convergents, bien que l'auteur adopte une autre chronologie. Levi déclare tout au début d'avoir eu la chance de n'être déporté à Auschwitz qu'en 1944 (SQU, 9). Son explication qui suit, concernant l'amélioration sensible des conditions de vie dans les camps qui visait à remédier aux problèmes avec la main-d'œuvre en Allemagne, ne laisse aucune trace d'émotion. D'un ton sec et froid il explique «sua fortuna ». Le bref rappel des événements lié à son statut du déporté lui permet en quelque sorte de passer à celui de l'écrivain-témoin. Levi expose son objectif, en soulignant qu'il ne cherche point à enrichir le savoir des lecteurs sur les camps de concentration, ni, ce qui le rapproche d'Antelme, à en accuser les responsables. «Esso non è stato scritto allo scopo di formulare nuovi capi di accusa ; potrà piuttosto fornire documenti per uno studio pacato di alcuni aspetti dell'animo umano " ( $\mathrm{SQU}, 9)$. Ce qu'il y a de remarquable dans cette proposition, ce n'est pas seulement qu'elle soit une incitation à faire de son témoignage un objet d'étude, mais qu'elle formule clairement la tâche qu'assigne cette parole sur les camps à ceux qui la reçoivent. Levi veut nous instruire par son écriture, tout en développant notre connaissance de certains aspects de l'âme humaine ${ }^{16}$. En même temps, il s'excuse auprès de ses futurs lecteurs de ses imperfections qu'il justifie par le fait que l'idée de son livre date de l'époque de sa déportation. A cela s'ajoute l'urgence d'écriture, mentionnée déjà plus haut. Le désir frénétique chez Antelme, et un impulso immediato e violento, comme l'appelle Levi, forcent en quelque sorte les deux survivants à prendre la plume et faire participer les autres à leur expérience.

En terminant sa préface, Levi pose une problématique intéressante. S'il a écrit son livre, c'est avant tout en vue d'une libération intérieure. Il se peut que derrière cette constatation se cache le vrai dessein du déporté qui témoigne de son vécu personnel. Il décide de faire connaître aux autres la vérité parce que par le biais de cet effort intellectuel, la mémoire encore vive des événements se trouve transformée. Ainsi, la rédaction de son témoignage, bien qu'elle l'oblige à revivre et repenser les souvenirs qu'il cherche à oublier, l'amène paradoxalement à se libérer du mal accumulé dans son âme et dans son esprit. L'écriture apparaît en l'occurrence comme une activité bienfaisante et cathartique pour les survivants et le récit même devient pour eux le moyen d'échapper à leur condition des morts-vivants. Les rescapés qui viennent témoigner représentent tous ceux, proches ou non, qui ne sont pas revenus des camps de concentration. Ils témoignent au nom des absents et

${ }^{16}$ A. Parrau parle en l'occurrence d'une certaine capacité subjective enracinée dans une éthique de la connaissance, la même qui, devenue principe d'une écriture, donne chance à sa valeur dc vérité. Cf. A. Parrau, Ecrire les camps, Belin, Paris 1995, p. 109. 
de leur mémoire ${ }^{17}$. C'est à ce titre que la vérité de l'expérience de l'auteur-témoin vaut comme argument d'autorité et implique la transmission de la vérité.

Dans les péritextes levien et antelmien retentit un projet littéraire que les deux écrivains se proposent de réaliser. Ils dépassent les conventions propres au témoignage au sens habituel, entendu comme un simple document, discours portant les traces du passé. Leur expérience singulière résonne tant à un niveau personnel que collectif, et se transforme en une réflexion universelle sur la déportation et le génocide. Ceci est particulièrement visible dans l'écriture antelmienne où le caractère inouï du vécu n'est jamais résorbé dans la familiarité d'une culture politique commune. Dans L'espèce humaine par exemple, la réflexion politique est abordée par le cheminement de l'expérience corporelle qui est la seule valable afin de mesurer la dimension de l'horreur concentrationnaire. Les divisions en classes ou en races n'importent donc point, puisqu'il est toujours question d'une seule espèce humaine :

(..) la variété des rapports entre les hommes, leur couleur, leurs coutumes, leur formation en classes masquent une vérité qui apparaît ici éclatante, au bord de la nature, à l'approche de nos limites : il n'y a pas des espèces humaines, il y a une espèce humaine (EH, 240).

L'auteur de L'espèce humaine exprime la déshumanisation du point de vue de l'intériorité. Elle est filtrée par la conscience agressée du «je» ou du «on», deux instances qui ne restent pas sans importance quant à la tonalité générale du texte :

On est entrés dans une salle minuscule et humide dans laquelle se trouvait une baignoire. Un civil en blouse blanche nous a donné des portemanteaux de fer. Los, los! On s'est vite déshabillés; on était malhabiles, on s'embrouillait dans nos ficelles. Tout était par terre, en vrac. J'ai accroché ma chemise ma veste, mon pantalon, les chiffons de mes pieds sur le portemanteau. J'étais nu (...) Il y avait de la boue par terre. On a décidé de se laver d'abord la figurc. Une pierre comme savon. On s'est mis à racler. Un jus noir coulait sur la poitrine couverte de croûtes. Très vite, l'eau de la baignoire s'est noircie. La figure était en feu, elle n'était pas encore propre mais on n'osait plus la tremper dans l'eau noire (...) je me suis mis à me racler les cuisses de toutes mes forces. Cuisses de vieillard, $j$ 'en faisais presque le tour avec ma main. La pierre, sèche, ne glissait pas sur la peau. Des rigoles d'eau noire couraient sur les plaies des jambes (EH, 128-129).

Ces phrases révèlent donc la caractéristique propre à l'écriture antelmienne qui consiste à traduire à travers le «on " l'oppression indistincte subie par tous les

${ }^{17}$ Quarante ans après sa déportation, P. Levi est amené à constater que les meilleurs (témoins) sont tous morts et il juge nécessaire de le souligner avec force, en mettant en question le statut de survivant-témoin : «Nous, les survivants, nous ne sommes pas les vrais témoins. (...) nous sommes ceux qui, grâce à la prévarication, l'habilité ou la chance, n'ont pas touché le fond. Ceux qui l'ont fait, qui ont vu la Gorgogne, ne sont pas revenus pour raconter, ou sont revenus muets, mais ce sont eux, les " musulmans », les engloutis, les témoins intégraux, ceux dont la déposition aurait eu une signification générale". Cf. P. Levi, Les naufragés et les rescapés. Quarante ans après Auschwitz (traduit de l'italien par A. Maugé) Gallimard, Paris 1989, p. 82. 
détenus et, respectivement, à désigner par le «je », un de ses éléments opprimés. Antelme raconte donc son histoire particulière même si cette histoire est celle de l'espèce humaine dans son ensemble. Ce n'est pas un regard qui se pose sur le monde et par lequel le lecteur peut voir le camp, c'est plus une présence qui permet de franchir les barbelés avec le narrateur et épouser sans aucune rupture sa vision, comme si l'univers du camp s'organisait par moments autour de cette seule personne :

Le soir, je suis allé au revier, dont Gilbert était devenu le secrétaire.(..) J'ai demandé de la schonung parce que je n'y voyais pas; le médecin m'a dit qu'il ne pouvait rien faire si je n'avais pas de fièvre. Gilbert lui a dit que j'étais un ami ; il a signé un billet de trois jours de schonung. D'autres sont passés, des Italiens surtout, plus épuisés que moi ; ils n'ont pas eu de schonung (EH, 175-176).

La première personne du singulier si fréquemment utilisée, propre à tout texte à caractère testimonial, acquiert ici une dimension particulière, puisqu'elle est cette conscience solitaire qui résiste et dont l'omniprésence organise et domine la structure de l'œuvre. Ce « je » fonctionne parfois implicitement dans le récit en sorte que le passage au «nous» ou «on» ne se fait pas beaucoup sentir. Le lecteur se déplace avec le narrateur sans vraiment prendre conscience de cette opération qui donne au destin individuel la dimension collective, comme dans le passage cidessous, du début du récit :

Je suis rentré dans le block parce qu'il n'y avait même pas de quoi rester dehors à regarder en l'air cette nuit-là. Il n'y avait rien dans le ciel, et sans doute il n'allait rien venir. Le block, c'était chez nous, notre maison. C'est là qu'on dormait, c'était là qu'un jour on avait fini par arriver. Je suis remonté sur ma paillasse (EH, 15-16).

Le « on " implique ici un ensemble contre l'oppresseur, il sert de protection contre la menace de la mort, enfin, permet de retrouver un peu d'espoir, parce qu'il désigne le destin égal auquel sont soumis tous les détenus, susceptibles parfois de résister encore.

Dès le début de Se questo è un uomo, on a une étrange impression d'assister, au fur et à mesure que le récit avance, à un parcours initiatique de l'auteur. $\mathrm{Si}$, au départ, les actions sont bien exécutées par ce « je » à travers lequel retentit l'histoire de "nous", désignant tous les déportés, la réflexion concernant le vécu est exprimée par une autre instance. Petit à petit, ce jeune homme sans expérience, peu capable, apparemment, de survivre dans un camp de concentration, subit une transformation. La réalité concentrationnaire forge l'auteur: la distance initiale entre Levi - auteur, qui avec un regard rétrospectif relate son entrée dans le camp et Levi - narrateur, qui lutte au quotidien contre la faim, le froid et la mort, se réduit. Le « je » de l'auteur et celui du narrateur se confondent ainsi dans la même voix :

Quest'anno è passato presto. L'anno scorso a quest'ora io ero un uomo libero; fuori legge ma libero, avevo un nome $\mathrm{c}$ una famiglia, possedevo una mente avida $\mathrm{e}$ inquieta $\mathrm{e}$ un corpo 
agile e sano. Pensavano a molte lontanissime cose: al mio lavoro, alla fine della guerra, al bene e al male, alla natura delle cose e alle leggi che governano l'agire umano (...) I miei giorni erano lieti e tristi, ma tutti li rimpiangevo, tutti erano densi e positivi; l'avvenire mi stava davanti come una grande ricchezza. Della mia vita di allora non mi resta oggi che quanto basta per soffrire la fame e il freddo; non sono più abbastanza vivo per sapermi sopprimere (SQU, 127).

Chez Levi, nous pouvons remarquer un phénomène intéressant qui consiste en un changement narratif, effectué au cours du récit. D'un chapitre à l'autre, Levi passe de la narration à la première personne au point de vue globalisant d'un savoir. Ce passage n'est pas sans rapport avec un "avancement" de l'auteur dans la hiérarchie concentrationnaire : du détenu condamné aux travaux physiques les plus durs il devient un employé de laboratoire, protégé des conditions extrêmes de l'oppression et capable en même temps de porter un regard plus lucide sur son vécu, visant en premier la compréhension et l'explication. Dans le laboratoire, Levi commence d'ailleurs à écrire et prendre les notes qui lui serviront plus tard à la rédaction de Se questo è un uomo. Cet acte de prélever alors sur le papier ses idées tćmoigne d'une certaine autonomie acquise par rapport aux oppresseurs. Par là, il réussit à arracher la connaissance à leur puissance et mème, il essaje de la retoumer contre eux.

La transcription de l'expérience effectuée par Levi repose donc sur deux dimensions, la première étant celle du point de vue de la connaissance et la seconde, le récit de sa propre expérience racontée au plus près. Quelle que soit la dimension, Levi ne renonce pas à l'affronter continuellement à travers la question de l'indicible, bien que, par moments, sa narration débouche sur un impossible à dire :

Allora per la prima volta ci siamo accorti che la nostra lingua manca di parole per esprimere questa offesa, la demolizione di un uomo (SQU, 23).

Ce qui caractérise son récit, c'est la recherche constante, entre la raison et l'écriture, d'une réponse convenable à la réalité du camp. Levi ne veut point céder devant ce produit créé par le système fasciste, et il ne le fait ni sur l'exigence de communiquer, ni sur celle de savoir, en cherchant à se frayer le chemin, en abordant les questions difficiles à transmettre, le jamais dit de la littérature de l'époque. Quand on le regarde de plus près, son récit est moins centré sur le moi que sur les multiples sujets de l'observation, tels effets traumatisants du système concentrationnaire, cas multiples de déshumanisation, naturalisme de l'étrange comportements et réactions des victimes. Il en résulte que le texte privilégie l'objet aux dépens du seul sujet, ce qui est également révélateur dans un témoignage. A cet égard, la démarche de $P$. Levi s'apparente à la démarche scientifique.

A cela s'ajoute un autre élément important, la présence des références culturelles auxquelles recourt l'auteur et qui lui permettent d'établir une relation entre l'expérience de l'horreur concentrationnaire et sa restitution par le biais de la littérature. Sur cela repose d'ailleurs l'originalité de Se questo è un uomo. Grâce à ces 
références, Levi - auteur procède à une transmission littéraire, et Levi - acteur, déporté réduit à sa dimension animale, échappe à sa condition de mort - vivant, mentionnée ci-dessus. Il fait ainsi preuve de sa capacité à communiquer, à penser, enfin à vivre. La culture, même si elle résulte plutôt d'un enseignement scolaire que de la vraie passion pour la littérature, joue pour le prisonnier un rôle important, devient par moments son soutient quotidien, indispensable à la survie ${ }^{18}$.

Les références en question forment un système cohérent à l'intérieur de l'œuvre levienne, ce qui est dû entre autres à leur source. Les passages que Levi insère dans son texte pour mieux exprimer son destin sont empruntés à Dante. Leur choix n'est pas fortuit, car ils se concentrent autour d'un thème principal qui est le voyage $^{19}$. Ce voyage peut être examiné d'abord dans l'optique d'un témoignage sur l'univers concentrationnaire, mais aussi dans une autre perspective, celle d'une interrogation morale sur Auschwitz. C'est le voyage de l'homme au fond de sa nature, ses limites, le "voyage - errance » qui exprime la recherche de sa propre identité, bousculée par l'événement concentrationnaire. Ceci étant, on pourrait analyser la question du déplacement soit dans sa dimension individuelle (l'auteur témoigne de son chemin), soit dans sa dimension collective, donc entendue comme l'expérience vécue par des milliers (les déportés) et suivie par des milliers (les lecteurs).

Si Levi décide d'emprunter à Dante ce thème du voyage c'est avant tout pour l'appliquer à sa réflexion sur le génocide. Dès le début on aperçoit dans son discours les parallèles narratifs entre l'Enfer dantesque et le camp ${ }^{20}$. Les deux

${ }^{18}$ Le rapport à la littérature, à Auschwitz, est écartelé cntre les deux impératifs contradictoires, comme l'observe A. Parrau : oubli ou mémoire, l'un et l'autre mettant en jeu la possibilité de survivre. D'une part Levi affirne que la culture, l'art et la poésie ne peuvent pas servir à comprendre la réalité du camp, puisque l'entreprise nazie est tout entière placée sous le signe de l'oubli (contraindre les détenus à "oublier» leur l'humanité). Ceci étant, il était salutaire. constate Levi, de les fla raison, l'art, la poésie] oublier, comme il était salutaire d'apprendre à oublier la maison et la famille. Or, il n'est pas question d'un oubli délinitif, mais d'une sorte de "relégation », selon le terme de Levi. D'autre part, il suftit de lire le tout petit chapitre de Se questo è un uomo, intitulé $/ /$ canto di Ulisse pour s'apercevoir qu'il jouit d'un statut rematquable par rapport à tout le récit, car il présente l'événement qui tranche absolument sur le quotidien du camp, constitue une sorte de parenthèse culturelle. Si l'on analysc encore l'application avec laquelle l'auteur reconstitue cetle scène où il se donne de la peine pour expliquer à son ami français un passage de Divine Comédie (tout en allant chercher la soupe de leur Kommando), la confirnation précédente, d'un nécessairc oubli de la littératurc, n'est plus valable. Son travail de remémoration (et de traduction) qu'il entreprend alors, traduit le mieux la capacité de la littérature à reconstituer la notion d'une communauté humaine. Cf. P. Levi, Les naufragés et les rescapés. Quarante ans après Auschwitz, op. cit, pp. 139-140, et A. Parra u, Ecrire les camps, op. cit., pp. $252-253,261-262$.

${ }^{19} \mathrm{~J} . \mathrm{Ph}$. Bareil consacre à l'étude de cette question tout son ouvrage intitulé Exil et voyage littéraire dans l'auvre de P. Levi, Ed. Messene, Paris 1998.

${ }^{20} \mathrm{H}$. Arendt y recourt également, en évoquant différentes formes de camps de concentration. L'atmosphère d'irréalité et de rêve qui règne dans le Lager, crééc par une apparente absence de but, conduit la philosophe à constater que, vus de l'extérieur, les camps ne peuvent être décrits qu'à l'aide 
chapitres qui ouvrent Se questo è un uomo, intitulés respectivement $l l$ viaggio et Sul fondo se réfèrent constamment à l'œuvre de Dante et déterminent déjà la structure du témoignage levien: l'arrivée au Lager, la découverte du système concentrationnaire et le processus de déshumanisation des prisonniers. L'ambiance qui accompagne ces événements, située entre le rêve et la réalité, semble confirmer la spécificité de cette nouvelle expérience. Le voyage même vers le camp est pour les déportés un voyage infernal et annonce leur destin :

Soffrivamo per la setc $\mathbf{c}$ il freddo : a tutte le fermate chiedevamo acqua a gran voce, $o$ almeno un pugno di neve, ma raramente fummo uditi (...) Nessuno tentava più, durante le soste, di comunicare col mondo esterno : ci sentivamo ormai «dall'altra parte " (SQU, 15, 16).

Une fois le convoi arrivé, les déportés découvrent petit à petit cet univers, clos et régi par ses propres règles, et dans le camion qui les emmène au canp, ils commencent à se rendre compte de ce qui les attend. C'est à ce moment - là, à la fin du premier chapitre, qu'apparaît la première citation tirée de la Divine Comédie, à travers laquelle Levi établit le parallèle direct entre le monde dantesque et concentrationnaire :

Troppo tardi, troppo tardi, andiamo tutti «giù ». D'altronde, ci siamo presto accorti che non siamo senza scorta : è una strana scorta. E un soldato tedesco, irto d'armi : non lo vediamo perché è buio fitto, ma ne sentiamo il contatto duro (...) Accende una pila tascabile, e invece di gridare "Guai a voi, anime prave " " ci domanda cortesemente ad uno ad uno, in tedesco $\mathrm{e}$ in lingua franca, se abbiamo danaro od orologi da cedergli : tanto dopo non ci servono più (SQU, 18).

Le chapitre suivant intitulé Sul fondo, riche également en analogies ou descriptions proprement dantesques, s'ouvre avec un épisode de la salle de douche. Cette scène à caractère initiatique apprend encore une fois aux déportés leur situation. Le narrateur est amené à constater :

Oggi, ai nostri giorni, l'inferno deve essere così, una camera grande e vuota, e noi stanchi stare in piedi, e c'è un rubinetto che gocciola e l'acqua non si può bere, e noi aspetiamo qualcosa di certamente terribile e non succede niente e continua a non succedere niente. Come pensare ? Non si può più pensare, è come essere già morti (SQU, 19).

Il convient de revenir encore sur la question des tonalités présentes dans les deux témoignages. L'ironie, l'humour ou le lyrisme des récits en question té-

d'images titrées d'une vie post mortem. Ainsi, elle distingue trois types de camps de concentration qui correspondent à trois conceptions fondamentales de la vie après la mort en Occident : Hadès, réunissant des éléments «indésirables » dans les pays non-totalitaires (réfugiés, asociaux, chômeurs, etc.), le Purgatoire, représenté par Jes camps de travail en Union soviétique ct l'Enfer, où, au sens littéral aussi, l'ensemble de la vie fut minutieusement et systématiquement organisée en vue des plus grands tourments. Cf. H. A rendt, les origines du totalitarisme. Le système totalitaire, Seuil, Paris 1995, pp. 182-183.

${ }^{21}$ Cettc expression, traduite en français par Henri Longnon comme "gare à vous, âmes noires" vient de la Divine Comédie de Dante, Enfer, chant troisième (cf. Classiques Garnier, Paris 1999). 
moignent également d'une capacité extraordinaire de leurs auteurs à dépasser le tragique par ce qui peut être plus fort encore dans l'évocation. C'est par exemple la manière de traduire la dégradation de l'homme et de sa mort. Selon Levi et Antelme, celle-ci s'inscrit dans le rythme de l'existence du Lager et n'est qu'un des ćléments de leur quotidien. Antelme, en évoquant par exemple la mort d'un copain parle d'une catastrophe. Or c'est une espèce de catastrophe qu'on oublie vite et qui se répète quasiment tous les jours, sans rien changer :

Ça ne fait pas de bruit, rien ne s'arrête. Il meurt, c'est l'appel, il meurt, c'est la soupe, il meurt, on reçoit des coups, il meurt seul (EH, 106).

A la lumière de la réflexion antelmienne, la mort n'est pas catastrophique en elle-même, ce sont les conditions dans lesquelles elle s'effectue qui sont inacceptables. Mourir dans la solitude, inaperçu, c'est ce qui est le plus dramatique aux yeux de l'auteur de L'espèce humaine. Néanmoins, chez Antelme, la mort, considérée comme un phénomène qui se produit à grande échelle dans la réalité concentrationnaire, peut acquérir, sur un autre plan, une fonction positive. Elle n'est pas un simple acte de résignation, mais une délivrance dans le sens où les fascistes ne peuvent plus réaliser leurs objectifs et poursuivre leur projet d'avilissement continuel de l'homme. "Plus on est contesté en tant qu'homme par le SS », constate le narrateur, «plus on a de chances d'être confirmé comme tel » $(\mathrm{EH}, 107)$.

On entend ici un écho de la thèse de Michel Foucault, qui conçoit la mort, conformément à sa vision de la bio politique moderne, comme "le moment où l'individu échappe à tout pouvoir, retombe sur lui-même et se replie en quelque sorte, sur sa part la plus privée $»^{22}$. C'est ainsi donc que Antelme entend sa philosophie de l'espèce humaine qui, contrairement à ce que soutient $\mathrm{P}$. Levi, se confirme justement au plus haut degré de la dégénérescence de l'homme. "Vous avez refait l'unité de l'homme », remarque l'auteur de L'espèce humaine (EH, 100), en s'adressant à un moment donné aux SS. Cette pensée est au cœur de son témoignage. L'homme devenu une présence anonyme, sans visage, privé de sa dignité essentielle d'homme et réduit à l'extrême dénuement, ressent pourtant le sentiment ultime d'appartenance à l'espèce, comme conclut Antelme. Sa singularité est sauvegardée malgré la transformation qu'il subit.

Tous les auteurs des témoignages parlent de la manière dont s'effectue la démolition de l'homme, mais $\mathrm{P}$. Levi et $\mathrm{R}$. Antelme l'illustrent d'une façon évocatrice. Petit à petit on suit avec eux leur propre déchéance physique et morale, leur abandon total par lequel ils se rapprochent de la figure du «musulman $»^{23}$. Bien que leur statut de témoin les autorise à parler à la première personne du singulier, ils

${ }^{22}$ M. Fouc a ult, Il fuut défendre la société, Gallimard, Paris 1997, p. 221.

${ }^{23}$ Dans le jargon du camp, ce terme désigne le détenu qui cesse de lutter, délaissć par ses camarades, un non-homme en qui l'étincelle divine s'est éteinte, comme ćcrit P. Lcvi. Pour voir l'origine et l'explication du terme cf. l'ouvrage de G. Agamben, Ce qui reste d'Auschwitz, Editions Payot \& Rivages, Paris 1999, pp. 49-57. 
décrivent rarement la dégradation de leur propre corps. Au « je », comme nous avons déjà vu plus haut, se superpose «nous » ou « on », représentant la masse de détenus. Il n'est pas forcément question d'une souffrance individuelle, mais d'un destin des milliers d'hommes qui traversent des étapes successives de transformation. Le canon esthétique traditionnel, opposant le beau et le laid, cesse d'exister : «On se transforme. La figure et le corps vont à la dérive les beaux et les laids se confondent $»(\mathrm{EH}, 97)$.

Si la description de certains personnages, ou de certains épisodes semblent relayer, par leur singularité, la tonalité plus grave, c'est souvent pour donner au camp une vision plus atroce et plus amère en même temps, comme ceci a lieu dans la description de la Bourse, proposée par Levi, où on peut échanger la chemise de réserve contre des produits de consommation et où les détenteurs se précipitent par exemple, pour y placer leur capital, en un moment de prospérité, comme dit l'auteur.

Le caractère dramatique de l'expérience vécue s'accorde avec sa transcription littéraire. Chez Levi, le récit semble divisé, comme au théâtre, en scènes durant lesquelles le temps se fige et la réalité inconcevable est décrite parfois à travers la terminologie théâtrale. Il «théâtralise » un peu son récit afin de mettre en abyme le côté irréel et absurde du camp, il découpe la réalité concentrationnaire en "actes" dont le déroulement est tant absurde qu'impossible :

Adesso è il sccondo atto. Entrano con la violenza quattro con rasoi, pennelli e tosatrici, hanno pantaloni e giacche a righe, un numero cucito sul petto (...). Noi facciamo molte domande, loro invece ci agguantano $\mathrm{e}$ in un momento ci troviamo rasi e tosati (...)

Ma ormai la mia idea c̀ che tutto questo è una grande macchina per riderc di noi e vilipenderci, e poi è chiaro che ci uccidono, che crede di vivere è pazzo (...)

Oramai siamo stanchi di stupirci. Ci pare di assistere a qualche dramma pazzo, di quei drammi in cui vengono sulla scena le streghe, lo Spirito Santo e il demonio (SQU, 20-21).

Ce ne sont que de petits exemples qui traduisent l'originalité des récits de Levi et d'Antelme. Bien que les différents auteurs des témoignages aient traversé des épreuves analogues et chacun ait apporté sa propre représentation de l'univers concentrationnaire, rares sont les récits qui méritent d'être rangés parmi les œuvres littéraires. Le rapport de ces deux textes à la littérature n'est donc pas dû à la pratique du genre littéraire, mais plutôt à leur caractère spécifique. La tonalité exceptionnelle de L'Espèce humaine et de Se questo è un uomo résulte surtout de la manière adoptée par les deux auteurs afin de rendre l'aspect non-représentable de leur expérience. Il s'avère que l'indicibilité langagière peut contribuer à la naissance d'une œuvre littéraire. Les textes d'Antelme et de Levi débordent leur vocation de témoignage, dépassent maintes fois les règles propres à ce genre voué d'emblée à la mémoire des camps et débouchent sur une réflexion profonde sur l'homme et sa nature. Ils sont en même temps l'expression de la transformation 
parfaite du vécu en langage et communiquent paradoxalement, pour reprendre ici la réflexion de Georges Perec appliquée à sa lecture de L'Espèce humaine, cette confiance illimitée dans l'écriture qui fonde toute la littérature :

La littérature commence ainsi, lorsque commence par le langage, dans le langage cette transformation (...) qui permet à un individu de prendre conscience, en exprimant le monde, en s'adressant aux autres ${ }^{24}$.

\section{BIBLIOGRAPHIE}

Agamben G. (1999), Ce qui reste d'Auschwitz, Bibliothèque Rjvages, Paris.

Antelme R. (1999), L'espèce humaine (EH), Gallimard, Paris.

A rend H. (1995), Le système totalitaire, Seuil, Paris.

Blanchot M. (1969), L entretien infini, Gallimard, Paris.

Cayrol J. (1953), Témoignage et littérature, Esprit, avril.

Dobbels D. (1999), Moncond'huy D. (dir.), Les camps et la littérature. Une littérature du XX siècle, La licorne, Poitiers.

Levi P. (1989), Se questo è un uomo (SQU), Einaudi, Torino.

Levi P. (2000), Les naufragés et les rescapés, Gallimard, Paris.

Parrau A. (1995), Écrire les camps, Belin, Paris.

Pipet L. (2000), La notion d'indicible dans la littérature des camps de la mort, L'Harmattan, Paris.

Riffaterre M. (1995), Le témoignage littéraire, Les Cahiers de la Villa Gillet, $n^{\circ} 3$, novembre.

${ }^{24} \mathrm{G}$. Perec, Robert Antelme ou la vérité de la littérature, dans : Robert Antelme. Textes inédits sur L'Espèce humaine. Essais et témoignages, Gallimard, Paris 2000, p. 190. 